\title{
Genetic correlation between autistic traits and IQ in a population-based sample of twins with autism spectrum disorders (ASDs)
}

Takeshi Nishiyama, Hiroko Taniai, Taishi Miyachi, Koken Ozaki, Makoto Tomita and Satoshi Sumi

Journal of Human Genetics (2009) 54, 191; doi:10.1038/jhg.2009.19

Journal of Human Genetics (2009) 54, 56-61; doi:10.1038/jhg.2008.3; published online 9 January 2009

The Author name Hiroko Taniai was published incorrectly in the above referenced paper. The correct author list is shown above.

The sentence mentioned below including the equation was also published incorrectly in the above referenced paper.

$$
L_{\bar{A}}=\int_{-\infty}^{t} \int_{-\infty}^{\infty} \int_{-\infty}^{\infty} \int_{-\infty}^{\infty} \varphi\left(x_{1}, y_{1}, x_{2}, y_{2} ; R\right) \mathrm{d} x_{1} \mathrm{~d} y_{1} \mathrm{~d} x_{2} \mathrm{~d} y_{2}
$$

where $x_{1}$ and $x_{2}$ are variables for integration over the normal distribution of CARS score for a proband and his co-twin, $y_{1}$ and $y_{2}$ are variables of IQ score for a proband and his co-twin, $\varphi$ is the fourvariate normal probability density function, $R$ is the correlation matrix (transformed from the covariance matrix), and $t$ is the threshold used for ascertainment.

The correct sentence is shown below.

$$
L_{\bar{A}}=\int_{-\infty}^{t} \int_{-\infty}^{\infty} \int_{-\infty}^{t} \int_{-\infty}^{\infty} \varphi\left(x_{1}, y_{1}, x_{2}, y_{2} ; R\right) \mathrm{d} x_{1} \mathrm{~d} y_{1} \mathrm{~d} x_{2} \mathrm{~d} y_{2}
$$

where $x_{1}$ and $x_{2}$ are variables for integration over the normal distribution of CARS score for individuals 1 and 2, $y_{1}$ and $y_{2}$ are variables of IQ score for individuals 1 and $2, \varphi$ is the four-variate normal probability density function, $R$ is the correlation matrix (transformed from the covariance matrix), and $t$ is the threshold used for ascertainment.

The corresponding author would like to apologize for these errors. 